\title{
FUENTES Y DISTRIBUCIÓN DE DIFERENTES TIPOS DE OBSIDIANAS EN SITIOS ARQUEOLÓGICOS DEL CENTRO-SUR DE CHILE (38-44S).
}

\author{
CHARLES R STERN*, CHRISTIAN GARCÍA**, XIMENA NAVARRO*** Y JORGE MUÑOZ ${ }^{* * * *}$
}

\begin{abstract}
RESUMEN
Fueron analizadas, por medio de la técnica ICP-MS, 35 muestras de obsidianas provenientes de dos sitios arqueológicos costeros (Chan Chan-18 al norte de Valdivia y Puente Quilo en Chiloé) y de seis sitios continentales de cazadores-recolectores (Alero Cabeza de Indio-1, Fundo Tres Arroyos-1, Flor del Lago-1, Los Riscos-1, Quillén-1 y Granaderos-2) del centro-sur de Chile, con el fin de determinar la composición química de elementos trazas para así poder compararlas con obsidiana de fuentes conocidas en la zona. Las obsidianas estudiadas son de diferentes colores e incluyen obsidiana negra (23 muestras), gris (3 muestras), translucida (5 muestras) y roja (4 muestras). De las 23 muestras de obsidiana negra, 3 tienen composición química que no corresponde a ninguna fuente de obsidiana conocida, mientras que las 20 restantes, que incluyen 2 muestras de Chan Chan-18 y 3 provenientes de Puente Quilo, son químicamente similares a la obsidiana negra (tipo MEL) de una fuente en los Nevados de Sollipulli. Las 3 muestras grises de Chan Chan-18 son semejantes a la obsidiana del Volcán Chaitén (tipo CH). Las 5 muestras translucidas y las 4 rojas son composicionalmente similar a obsidiana tipo PC1 que ocurre como rodados fluviales transportados a lo largo del Arroyo Covunco al este de la Cordillera Andina, en Neuquén central, Argentina. Sin embargo, esta obsidiana PC1 podría tener una fuente primaria más cercana a la divisoria de aguas en los Andes en el cinturón volcánico que se extiende al sureste desde la Caldera Copahue pasando por Pino Hachado hasta la Caldera Palao Mahuida y el cono Queli Mahuida inmediatamente al norte del Paso de Icalma. Este es el paso fronterizo que tiene la menor altura en la zona (1298 msnm) y pudo ser el lugar por donde circuló esta obsidiana hacia el occidente. El hecho que todas las muestras derivadas desde la relativamente remota $(>70 \mathrm{~km})$ fuente de obsidiana PC1 sean visualmente distintivas, translucidas y rojas, sugiere que estas obsidianas de colores distintivos presentaban mayor valor estético en comparación con la obsidiana negra MEL, más común y de origen más local $(<30 \mathrm{~km})$ en los Nevados de Sollipulli.
\end{abstract}

PALABRAS CLAVES: Obsidiana, centro-sur de Chile, sitios arqueológicos.

\footnotetext{
Department of Geological Sciences, University of Colorado, Boulder, CO 80309 USA. email Charles.Stern@colorado.edu * Área de Arqueología, Facultad de Estudios del Patrimonio Cultural, Universidad Internacional SEK, Santiago, Chile. email cuvieronius@gmail.com

*** Escuela de Antropología, Universidad Católica de Temuco, Temuco, Chile. email ximenavaharris@gmail.com

**** SERNAGEOMIN, Oficina Técnica Puerto Varas, Chile. email jmunoz@sernageomin.cl
} 


\section{SOURCES AND DISTRIBUTION OF DIFFERENT TYPES OF OBSIDIAN FROM ARCHAEOLOGICAL SITES IN CENTRAL-SOUTH CHILE (38-44º $)$.}

\section{ABSTRACT}

Thirty-five samples of obsidian from two coastal sites (Chan Chan-18 north of Valdivia and Puente Quilo in Chiloé) and six inland terrestrial-hunter gatherer sites (Alero Cabeza de Indio-1, Fundo Tres Arroyos-1, Flor del Lago-1, Los Riscos-1, Quillen-1 and Granaderos-2) in south-central Chile were analyzed, by ICP-MS techniques, to determine their trace-element chemistry for comparison with obsidian from know source in the area. The obsidian samples differ in color and include black (23 samples), grey (3 samples), translucent ( 5 samples) and red (4 samples) types. For the 23 black samples, 3 have chemistry that does not correspond to any know source, while the other 20, including 2 samples from Chan Chan-18 and all 3 from Puente Quilo, are chemically similar to black obsidian (MEL) from a source in the Nevados de Sollipulli. Three grey samples from Chan Chan-18 are similar to obsidian from Chaitén volcano $(\mathrm{CH})$. The 5 translucent and 4 red samples are all similar to each other and to type PC1 obsidian that occurs as fluvial transported cobbles along Arroyo Covunco east of the Andean Cordillera in central Neuquén, Argentina. However, this obsidian may have its primary source closer to the Andean drainage divide in the volcanic belt that extend southwest from Copahue caldera through Pino Hachado to Palao Mahuida caldera and Queli Mahuida cone just north of Paso de Icalma. This is the lowest elevation pass (1,298 $\mathrm{m}$ ) in this area and the probable path for the entrance of this obsidian form Argentina into Chile. The fact that all the samples derived from the relatively remote PC1 obsidian source $(>70 \mathrm{~km})$ are visually distinctive translucent and red suggest that this distinctly colored obsidian may have had some aesthetic value relative to the more local $(<30 \mathrm{~km})$ and more easily accessible black MEL obsidian from Nevados de Sollipulli.

KEY WORDS: Obsidian, south-central Chile, archaeological sites.

\section{INTRODUCCIÓN}

Las fuentes de obsidianas conocidas del centro-sur de Chile (Fig. 1) incluyen el domo ancestral de obsidiana dentro del cráter del Volcán Chaitén (Stern et al., 2002), el cual fue destruido y reemplazado por un nuevo complejo de domos durante la reciente erupción de este volcán iniciada en Mayo del 2008 (Muñoz et al., 2008), y un domo en las rocas del Plioceno-Pleistoceno en los Nevados de Sollipulli (Stern et al., 2008). La obsidiana de Chaitén $(\mathrm{CH})$ es, generalmente, de color gris con un porcentaje pequeño pero notable de cristales de feldespato de plagioclasa, mientras que la obsidiana de los Nevados de Sollipulli (MEL) es negra y libre de cristales o con escasos cristales muy pequeños.

En este trabajo presentamos análisis químicos de 35 obsidianas de diferentes colores provenientes de distintos sitios arqueológicos ubicados en el centro-sur de Chile, que incluyen dos sitios a lo largo de la costa y seis sitios al interior, en la Depresión Central y en el margen occidental de la Cordillera Andina Principal (Tabla 1). Las muestras de obsidianas son de variados colores tales como negro, gris o rojo, además de translucida. Estudios anteriores efectuados en los sitios costeros de Chan Chan-18 y Puente Quilo demostraron que las obsidianas grises provenían del volcán Chaitén, pero las otras obsidianas en estos sitios, de color negro, eran de fuentes desconocidas (Stern et al., 2002). En este trabajo concluimos que la fuente de estas obsidianas negras se ubica en los Nevados de Sollipulli. También, demostramos que la mayoría de las muestras de obsidianas negras encontradas en los sitios interiores de la Depresión Central fueron derivadas de los Nevados de Sollipulli, mientras que las obsidianas translucidas y rojas provienen de una fuente distinta situada al este de la Cordillera Andina Principal, en Neuquén, Argentina. 


\section{DESCRIPCIÓN DE SITIOS}

Quillén-1

Se ubica dentro de la depresión intermedia de Chile, en la Región de La Araucanía, en el fundo la Rinconada de Perquenco, al norte de la ciudad de Lautaro y al este del pueblo de Galvarino (Fig. 1). El sitio se localiza a 3925'S y $72^{\circ} 35^{\prime} \mathrm{W}$ (Tabla $1 \mathrm{~A})$, y se encuentra cercano a la confluencia de los ríos Quillén y Perquenco.
Corresponde a un alero que está en un valle llamado El Teatro, que toma el nombre de su particular geomorfología. El valle está formado por un complejo de aleros y cuevas pequeñas, cercanas unas de otras, que conforman un verdadero teatro.

A esta latitud, los materiales que rellenan la Depresión Central están formados por depósitos laháricos ricos en rodados basálticos de grano fino (afaníticos), fuertemente cementados por ceniza volcánica y material calcáreo. En el alero Quillén1 , así como en los vecinos a éste, hay estalactitas

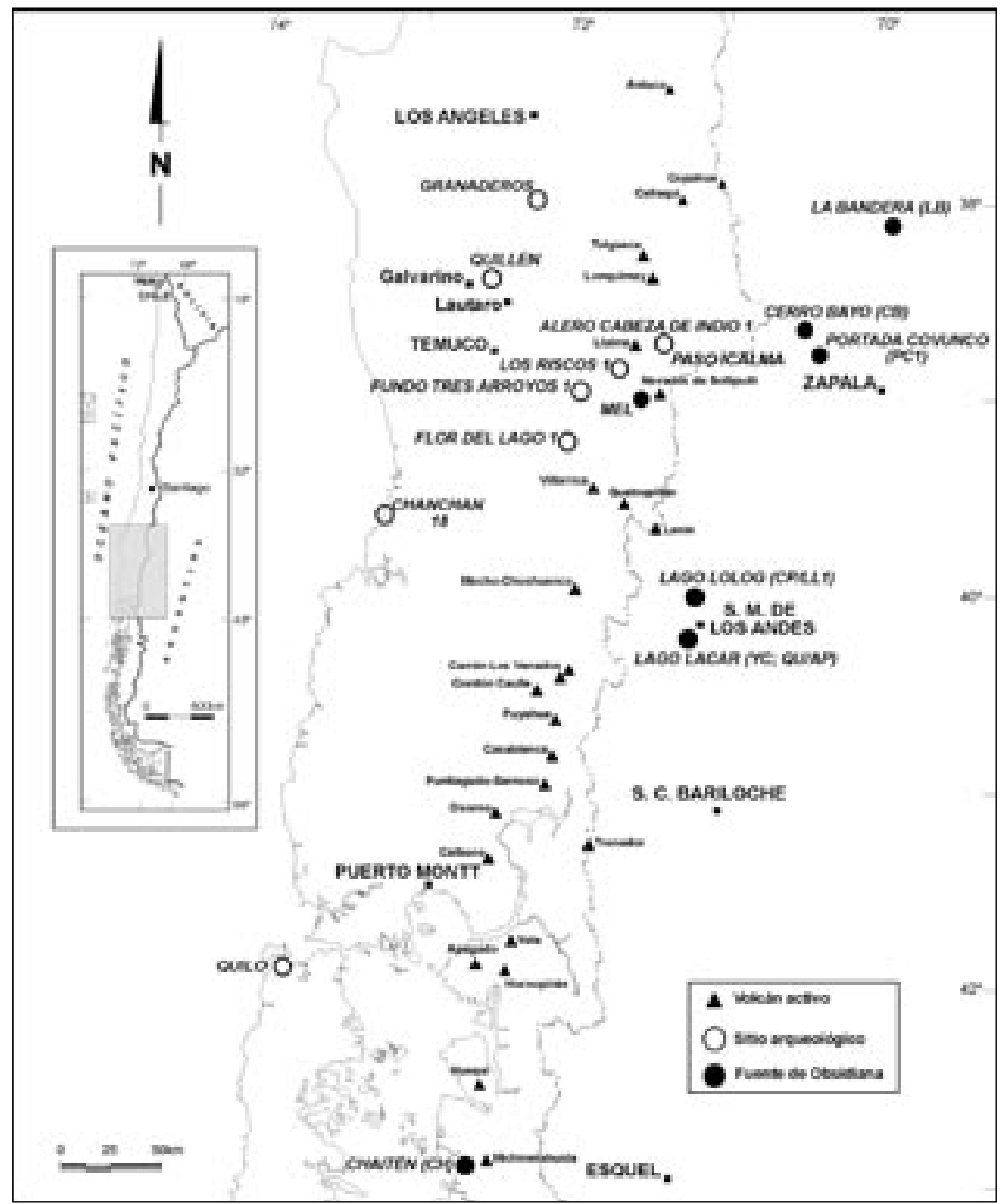

Fig. 1. Mapa de ubicación de los sitios arqueológicos del centro-sur de Chile de los cuales provienen los artefactos de obsidiana analizados Tabla 1A) y de las fuentes primarias y secundarias de obsidiana en Chile y Argentina en latitudes similares. La fuente de los distintos tipos de obsidianas incluyen: CH del Volcán Chaitén (Stern et al., 2002); MEL de los Nevados de Sollipulli al sur de Melipeuco (Stern et al., 2008); YC y QU/AP al norte de la costa del Lago Lácar (López et al., 2009); CP/LL1 del Cerro Planicies y del norte de la Costa del Lago Lolog (López et al., 2009); PC1 de Portada Covunco (Bellelli et al., 2002 y 2006; López et al., 2009); CB de Cerro Bayo (Stern, datos inéditos); LB de La Bandera. 
Tabla 1A. Sitios arqueológicos donde se recolectaron las obsidianas (analizadas) en este trabajo.

\begin{tabular}{|c|c|c|c|c|}
\hline Sitio & Latitud Sur & Longitud Este & Elevación & No. de muestras \\
\hline Alero Cabeza de Indio 1 & $-38^{\circ} 45^{\prime} 60^{\prime \prime}$ & $-71^{\circ} 33^{\prime} 57^{\prime \prime}$ & $842 \mathrm{msnm}$ & 15 \\
\hline Fundo Tres Arroyos 1 & $-38^{\circ} 58^{\prime} 47^{\prime \prime}$ & $-72^{\circ} 01^{\prime} 45^{\prime \prime}$ & $410 \mathrm{msnm}$ & 3 \\
\hline Flor del Lago 1 & $-39^{\circ} 12^{\prime} 20^{\prime \prime}$ & $-72^{\circ} 07^{\prime} 34^{\prime \prime}$ & $197 \mathrm{msnm}$ & 1 \\
\hline Los Riscos 1 & $38^{\circ} 53^{\prime} 05^{\prime \prime}$ & $-71^{\circ} 50^{\prime} 34^{\prime \prime}$ & $463 \mathrm{msnm}$ & 4 \\
\hline Quillen 1 & $-38^{\circ} 25^{\prime}$ & $-72^{\circ} 35^{\prime}$ & & 2 \\
\hline Granaderos-2 & $-37^{\circ} 59^{\prime}$ & $-72^{\circ} 15^{\prime}$ & & 1 \\
\hline Chan Chan-18 & $-39^{\circ} 34^{\prime}$ & $-73^{\circ} 16^{\prime} 54^{\prime \prime}$ & $12-19 \mathrm{msnm}$ & 6 \\
\hline Puente Quilo & $-41^{\circ} 51^{\prime} 36^{\prime \prime}$ & $-73^{\circ} 59^{\prime}$ & $2-4 \mathrm{msnm}$ & 3 \\
\hline
\end{tabular}

formadas por redepositación del material calcáreo presente en el cemento del lahar, que además cubre con una pátina importante los artefactos líticos expuestos.

El alero mismo mide $16 \mathrm{~m}$ de largo por $5 \mathrm{~m}$ de profundidad máxima y cerca de $2 \mathrm{~m}$ de altura. El sitio fue descubierto por un campesino que reconoció material cultural en su superficie y hasta ahora ha sido el único sitio excavado dentro del complejo de aleros del valle. La mayoría de ellos permanecen cubiertos por una vegetación densa que no permite observar sus entradas.

Quillén-1 fue excavado durante el año 1980, abriéndose seis (6) cuadrículas de $1 \mathrm{~m}^{2}$, en las cuales se diferenciaron dos áreas, una A conformada por 4 cuadrículas y una B por solo 2 cuadrículas, esta última ubicada al fondo del alero. El material cultural fue recuperado mediante separación de niveles artificiales de $10 \mathrm{~cm}$, hasta llegar a la capa estéril localizada a una profundidad de $120 \mathrm{~cm}$.

El estudio estratigráfico reveló que Quillén-1 tuvo al menos tres períodos distintivos de ocupación humana (Tabla 1B): uno temprano precerámico fechado en $4.675 \pm 105$ años AP; un segundo perteneciente al precerámico tardío datado en $2.030 \pm 70$ años AP y un tercero alfarero (Valdés et al., 1982). Los dos primeros están conformados únicamente por material lítico de basalto y algunos artefactos y desechos de obsidiana, calcedonia y jaspe. Los niveles alfareros se ubicaron entre la superficie y los $30 \mathrm{~cm}$ de profundidad. Como se excavó por niveles arbitrarios, lo más probable es que la fecha mas temprana represente la ocupación media del precerámico y no la edad más antigua de la ocupación (Valdés et al., 1982; Navarro, 1984).

Navarro (1984) analizó el universo lítico precerámico recolectado en el sitio Quillén-1, que estuvo compuesto por un total de 18.174 piezas entre artefactos, derivados de núcleos y lascas, y desechos de talla. Navarro (1984) estudió todos los ejemplares formatizados que se diferenciaron en los siguientes tipos: 225 núcleos, 1269 lascas modificadas y 23 puntas de proyectil. De este universo, el 99,2\% del total corresponde a material lítico de basalto local,

Tabla 1B. Fechas ${ }^{14} \mathrm{C}$ convencional (años A.P.) en los sitios estudiados.

\begin{tabular}{|c|c|c|c|c|c|}
\hline Sitio & Material & Método & Muestra & Fecha (años AP) & Referencia \\
\hline Chan Chan 18 & carbón & AMS & Beta-70189 & $5.320 \pm 150$ & Navarro y Pino 1995 \\
\hline Chan Chan 18 & carbón & AMS & Beta-89284 & $5.340 \pm 80$ & Navarro y Pino 1999 \\
\hline Chan Chan 18 & carbón & AMS & Beta-81423 & $5.000 \pm 70$ & Navarro y Pino 1999 \\
\hline Chan Chan 18 & carbón & Convencional & Beta-100572 & $5.070 \pm 130$ & Navarro y Pino 1995 \\
\hline Chan Chan 18 & carbón & Convencional & Beta-100573 & $5.180 \pm 150$ & Navarro y Pino 1995 \\
\hline Chan Chan 18 & carbón & Convencional & Beta-100574 & $5.360 \pm 90$ & Navarro y Pino 1995 \\
\hline Chan Chan 18 & carbón & Convencional & Beta-103555 & $5.460 \pm 50$ & Navarro y Pino 1995 \\
\hline Chan Chan 18 & carbón & Convencional & Beta-107499 & $5.610 \pm 100$ & Navarro y Pino 1995 \\
\hline Quillén 1 & carbón & Convencional & Beta-4710 & $4.675 \pm 105$ & Valdés et al. 1982 \\
\hline Quillén 1 & carbón & Convencional & Beta-4709 & $2.030 \pm 70$ & Valdés et al. 1982 \\
\hline Cabeza de Indio-1 & carbón & AMS & Beta-241263 & $670 \pm 40$ & García 2008 \\
\hline Cabeza de Indio-1 & carbón & AMS & Beta-241264 & $1.100 \pm 40$ & García 2008 \\
\hline Cabeza de Indio-1 & carbón & AMS & Beta-253959 & $1.830 \pm 40$ & García 2008 \\
\hline Flor del Lago-1 & carbón & AMS & Beta-253960 & $2.110 \pm 40$ & García 2008 \\
\hline
\end{tabular}


el que fue recolectado en los depósitos laháricos, mientras que solo un $0,8 \%$ corresponde a otras materias primas de origen alóctono tales como obsidiana negra, calcedonia, jaspe y esquisto gris.

Dos muestras de obsidiana negra provenientes de este sitio fueron analizadas. Ambas muestras son de niveles superficiales, o sea del período alfarero. Además, una muestra de obsidiana negra obtenida en la superficie del sitio de Granaderos-2, al norte de Quillén (Fig. 1; Tabla 1A), fue analizada. Granaderos-2 es un sitio superficial el cual fue descubierto en una prospección de impacto ambiental. En Granaderos-2 no existe una dispersión continua de material, sino una mezcla de material cerámico y lítico en un radio entre 100 por $150 \mathrm{~m}$.

\section{Cabeza de Indio-1}

El sitio arqueológico Alero Cabeza de Indio-1 se encuentra ubicado en la zona cordillerana de la provincia de Cautín, en la IX Región de La Araucanía, frente a los faldeos orientales del volcán Llaima (Fig. 1), a una altura de $842 \mathrm{msnm}$ en el valle del río Trufultruful (38 $45^{\prime} \mathrm{S}$ y $71^{\circ} 33^{\prime} \mathrm{W}$; Tabla $\left.1 \mathrm{~A}\right)$. Se excavaron tres unidades de $1 \mathrm{~m}^{2}$ siguiendo la estratigrafía y morfología natural del suelo, trabajos que se han llevado a cabo en dos campañas durante diciembre de 2007 y diciembre de 2008.

Las excavaciones en este sitio han documentado ocupaciones de cazadores recolectores portadores de cerámica asignables al complejo cultural Pitrén, así como otras más tempranas con ausencia de cerámica (Tabla 1B). Estas últimas han sido fechadas en $1.830 \pm 40$ años AP (Beta-253959) (García, 2008), en la base de la secuencia cultural a $120 \mathrm{~cm}$ de profundidad. Estas ocupaciones se caracterizan por una señal arqueológica débil, relacionada con escasas lascas de basalto, semillas quemadas de lleuque (Prumnopitys andina) y araucaria (Araucaria araucana), así como con carbones dispersos de fogones muy discretos. Posteriormente, a menor profundidad, la señal arqueológica en el alero es intensa, como consecuencia de recurrentes ocupaciones de grupos de cazadores recolectores portadores de cerámica. Dos de estas ocupaciones, asociadas a fogones y localizadas en profundidades diferentes, han sido datadas en $1.100 \pm 40$ años AP (Beta-241264) y $670 \pm 40$ años AP (Beta-241263) (García 2008). En general, ambas se asocian con cerámica monocroma, mientras que una pequeña fracción de piezas presenta diferentes tipos de decoración, entre las que se incluyen el ahumado, engobado, inciso, modelado y pintado. Entre las piezas decoradas destacan aquellas con "bandas modeladas paralelas" o "acanalada" (Hajduk, 1986), la cuales se presentan tanto en cuerpos como en cuellos de vasijas, tratándose mayoritariamente de fragmentos pulidos. Su importancia radica en que una técnica similar ha sido registrada en sitios arqueológicos del sector oriental de Los Andes, en la provincia de Neuquén (Fernández, 1988-90; Goñi, 1991; Hajduk, 1986). Igualmente, en Cabeza de Indio, se han registrado dos tubos de pipas, uno de ellos con una incisión en todo el diámetro de la boquilla, y cuentas de conchas, elementos que también se han registrado en el sector oriental. Además, se registra la presencia de puntas de proyectil de morfología triangular, perforadores y raspadores confeccionados con materias primas líticas de alta calidad, como obsidiana y sílices en diversidad de colores, junto con el uso del basalto local. A esta industria, se suman restos óseos de Lama guanicoe, Pudu pudu, valvas de Diplodón chilensis y piñones de araucaria (Araucaria araucana) carbonizados.

Otras ocupaciones más tardías presentes en el alero no han sido aún datadas, pero su asociación con fragmentos cerámicos con decoración de pintura roja sobre blanco, permite relacionarlos con la denominada Tradición Bícroma (Adán et al., 2005), que se expresa durante los últimos siglo después de la llegada de los españoles a la zona.

Quince muestras de obsidiana provenientes de este sitio fueron analizadas, incluyendo 7 de color negro, 4 de color rojo y 4 translúcido o transparente. Todas las muestras de Alero Cabeza de Indio-1 provienen del estrato 6 que tiene dos fechas (Tabla 1B): en la zona intermedia de $670 \pm 40$ años AP y en la base de $1.100 \pm 40$ años AP.

\section{Flor del Lago-1}

El sitio Flor del Lago-1 se localiza en la comuna de Villarrica, Provincia de Cautín, específicamente en la costa Norte del lago Villarrica (Fig. 1) a una altura de 197 msnm. Este sitio abierto se emplaza en una antigua terraza lacustre cubierta por un bosque maduro y a corta distancia de la costa del lago $\left(39^{\circ} 12^{\prime}\right.$ 'S y $72^{\circ} 07^{\prime} \mathrm{W}$; Tabla $\left.1 \mathrm{~A}\right)$. Fue reconocido en 
el lecho de un pequeño arroyo donde se observó abundante material cultural, particularmente cerámico y lítico, que destacaba por un tamaño de fragmentación relativamente alto. Este sitio fue sondeado y excavado, a raíz de lo cual se pudieron definir dos componentes ocupacionales. Uno temprano, datado en $2.110 \pm 40$ años A.P. (Beta-253960; Tabla 1B), el cual se caracteriza por fragmentos cerámicos monócromos pulidos y alisados, sin decoración, de paredes delgadas y artefactos líticos confeccionados en basalto local y obsidiana negra. Otro componente ocupacional, aún cuando no ha sido datado, tiene una apariencia más tardía debido a las características del material cerámico, tales como pintura roja sobre blanco, pastillaje, protuberos en el asa, entre otros, que lo vincularían a la Tradición Bícroma (Adán et al., 2005). De este sitio se analizó una muestra de obsidiana translúcida que proviene del estrato fechado en $2.110 \pm 40$ años A.P.

\section{Fundo Tres Arroyos-1 y Los Riscos-1}

El sitio arqueológico Los Riscos-1 se localiza en la provincia de Cautín, IX Región de la Araucanía (Fig. 1), sobre una antigua terraza fluvial del río Allipén, a 463 msnm, rodeada de cordones de cerros hoy reforestados con especies intrusivas (38 $53^{\prime}$ 'S. y $71^{\circ} 50^{\prime} \mathrm{W}$; Tabla 1A). Este sitio fue descubierto a través de una prospección realizada en junio de 2008 y fue reconocido por la presencia de abundantes materiales culturales en la superficie. Entre estos, se destacan fragmentos cerámicos monocromos, con paredes gruesas y delgadas, así como presencia de engobe rojo, artefactos líticos confeccionados en basalto y lascas de obsidiana negra, gris y cuarzo. Este sitio no ha sido sondeado y su cronología se relaciona al Holoceno tardío. Fueron analizadas cuatro muestras de obsidianas negras provenientes de este sitio.

El sitio Fundo Tres Arroyos-1 se localiza, también, en la provincia de Cautín en la IX Región de La Araucanía (Fig. 1), en la cuenca del lago Colico. Específicamente el sitio esta emplazado en un sector de lomajes suaves en el valle fluvial del río Allipén a una altura de $410 \mathrm{msnm}\left(38^{\circ} 58^{\prime} \mathrm{S}\right.$ y $72^{\circ} 01 ' \mathrm{~W}$; Tabla $\left.1 \mathrm{~A}\right)$. Este sitio, que no ha sido excavado ni datado, se relacionaría a grupos alfareros del Holoceno tardío, caracterizados por fragmentos cerámicos monocromos y lascas de basalto local y obsidiana. Tres muestras de obsidianas negras fueron analizadas.

\section{Chan Chan-18}

El complejo de sitios arqueológicos de Chan Chan se ubica a lo largo de la costa Pacífica cerca de Punta Chanchán, Región de Los Ríos, aproximadamente $30 \mathrm{~km}$ al norte de Valdivia (Fig. 1; Navarro y Pino, 1995, 1999). El sector donde se ubica el sitio de Chan Chan-18 y otros sitios arcaicos al norte de Valdivia se extiende en una franja costera de $30 \mathrm{~km}$ de longitud por $2 \mathrm{~km}$ de ancho, que incluye además las localidades de Maiquillahue, Alepúe, Puerto Nuevo y Pelluco, en la cual se han registrado un total de nueve sitios arcaicos. Estos sitios representan un patrón espacial de ocupación de bordemar en el límite de la playa o berma en terrazas litorales muy bajas (entre 9 y $12 \mathrm{msnm}$ ). El registro arqueológico de estas ocupaciones ha sido perturbado por drásticos procesos postdepositacionales, cómo fue aquel del último tsunami asociado al terremoto de 1960 . No obstante, los sitios reúnen condiciones de habitabilidad ideales y la visibilidad desde ellos es excelente, tanto hacia el océano Pacífico como hacia la zona de la Cordillera de la Costa. Tanto las penínsulas cómo las playas disipativas ofrecieron un acceso fácil a recursos marinos de roqueríos, al estuario y a lagunas costeras, en su mayoría hoy secas.

El sitio más extenso y potente es el de Chan Chan-18, el que además posee distintas áreas de actividad (doméstica, talleres líticos, de faenamiento y funebria). Este sitio fue ocupado al menos hace 5610 años A.P. (Navarro y Pino, 1999). La distribución de los fechados radiocarbónicos con respecto a la profundidad de excavación permite demostrar dos períodos de ocupación; el primero correspondiente a las fechas radiocarbónicas entre 5.460 y 5.610 años A.P. y el segundo entre 5.000 y 5.360 años A.P., estando ambos conjuntos están separados por un hiatus estratigrafico de ocupación.

El material lítico de este sitio es abundante y está conformado por lascas en sus distintas fases, desechos de talla, preformas y puntas foliáceas de tradición andina. Durante su excavación, se recuperaron ejemplares líticos, divididos en artefactos y desechos de talla. En superficie, se recolectó otra cantidad similar de artefactos y de ejemplares líticos en 
proceso de elaboración. La mayoría del instrumental lítico de estos cazadores recolectores arcaicos fue manufacturada en basalto y otras materias primas locales, tales como cuarzo, cuarcita, serpentinita, pizarra y esquisto. Con menor incidencia aparecen instrumentales de rocas andinas, tales como obsidiana y riolita gris.

En un estudio anterior fue demostrado que los artefactos de obsidiana gris encontrados en Chan Chan-18 provenían del Volcán Chaitén, aproximadamente $400 \mathrm{~km}$ al sur (Stern et al., 2002). En el presente trabajo se analizaron tres nuevas muestras de obsidiana gris y tres de obsidiana negra.

Puente Quilo

El sitio arqueológico de Puente Quilo se ubica en el golfo de Quetalmahue de la Isla Grande de Chiloé, aproximadamente $15 \mathrm{~km}$ al oeste de Ancud (Fig. 1), en la ribera oeste del río Quilo inmediatamente al sur del puente del mismo nombre. Las investigaciones arqueológicas, efectuadas mediante excavaciones realizadas el año 2000, indicaron que el nivel más antiguo de ocupación alcanzado se ubica alrededor de 5.030 años A.P., aunque no se descarta que edades más antiguas se obtengan por debajo del nivel anterior o en otros sectores del mismo sitio aún no explorados. En el sitio se verificó la presencia de huesos de lobo marino asociados a artefactos líticos, unifaciales y bifaciales, tales como bifaces de doble punta (Fig. 2), puntas foliáceas, lanceoladas y triangulares, entre otros, representativos de un campamento donde se trabajó en un taller lítico, además de entierros de humanos y presencia de abundante restos de carbón. Aunque los restos de conchas de bivalvos están escasamente representados en los niveles inferiores, los superiores corresponden a un basural conchífero.

Trabajos previos (Stern et al., 2002) concluyeron que la fuente de las muestras de obsidiana gris de este sitio estaba del Volcán Chaitén y que la fuente de dos puntas de obsidiana negra era desconocida. Estas dos puntas negras y una tercera (Fig. 2) fueron nuevamente analizadas en el presente estudio.

\section{MÉTODOS ANALÍTICOS}

Las muestras de obsidianas fueron pulverizadas para su posterior disolución en una solución con HF diluido. Una vez en solución, las muestras fueron analizadas por medio de técnicas ICP-MS. Los repetidos análisis de los estándares internos sugirieron que la precisión de los resultados es de $\pm 10 \%$ en a los niveles de concentración presentados por los diferentes tipos de obsidianas.

\section{Quilo, Isla Grande de Chiloé}

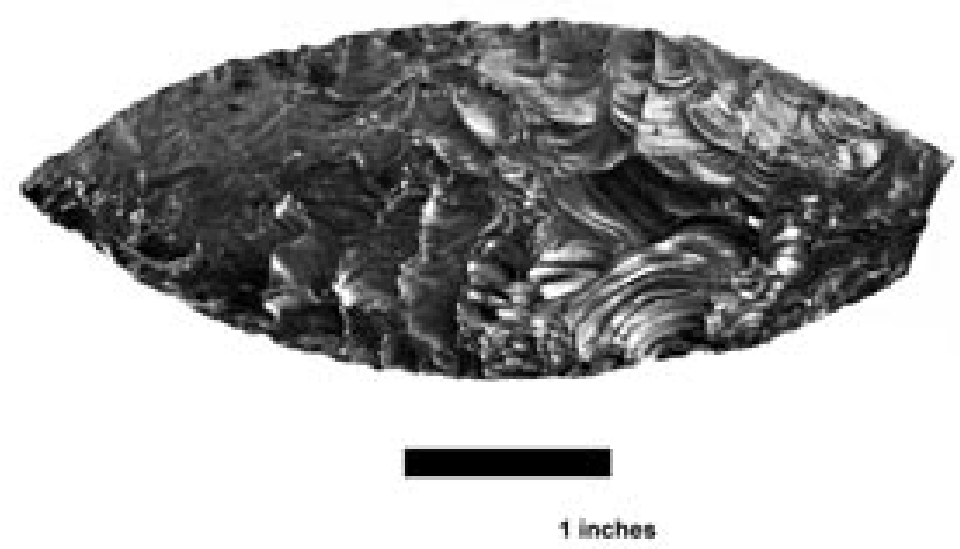

Fig. 2. Foto del artefacto de obsidiana bifacial de doble punta de Puente Quilo analizada en este estudio (Tabla 5). 


\section{RESULTADOS}

Tres de las obsidianas negras analizadas, una de Quillén-1, otra de Granaderos-2 y una de Chan Chan-18, tienen composición química que no corresponde con ninguna fuente conocida en Chile o Argentina (Fig. 3). Estas tres obsidianas negras tienen un contenido muy alto de $\mathrm{Zr}$ y de otros elementos de alto potencial iónico (HFSE; $\mathrm{Ti}, \mathrm{Y}, \mathrm{Hf}, \mathrm{Pb}$; Tablas 4 y 5). La presencia de este tipo de obsidiana en los dos sitios localizados más al norte sugiere que esta obsidiana podría haber provenido de una fuente ubicada aún más al norte y aún desconocida.

Todas las otras 20 muestras de obsidianas negras analizadas en este estudio, incluyendo las 7 muestras de Alero Cabeza de Indio-1 (Tabla 2),
1 de Quillén-1 (Tabla 4), 4 de Los Riescos-1 (Tabla 4), 3 de Fundo Tres Arroyos-1 (Tabla 4), 2 de Chan Chan-18 (Tabla 5) y las 3 puntas de Puente Quilo (Tabla 5) son similares a la obsidiana MEL de la fuente en los Nevados de Sollipulli (Fig. 3 y Tabla 6; Stern et al., 2008).

Las tres muestras de obsidiana gris de Chan Chan-18 son similares a la obsidiana $\mathrm{CH}$ del Volcán Chaitén (Tabla 5), lo cual ya había sido demostrado anteriormente (Fig. 3 y Tabla 6; Stern et al., 2002).

Cinco muestras de obsidiana translucida y cuatro de obsidiana roja (Tabla 3), todas, excepto una, del sitio Alero Cabeza de Indio-1 tienen composición química distintiva y son, tentativamente, clasificadas como obsidiana Alero Cabeza de Indio tipo 1 (ACI1). Otra muestra translucida tiene con-

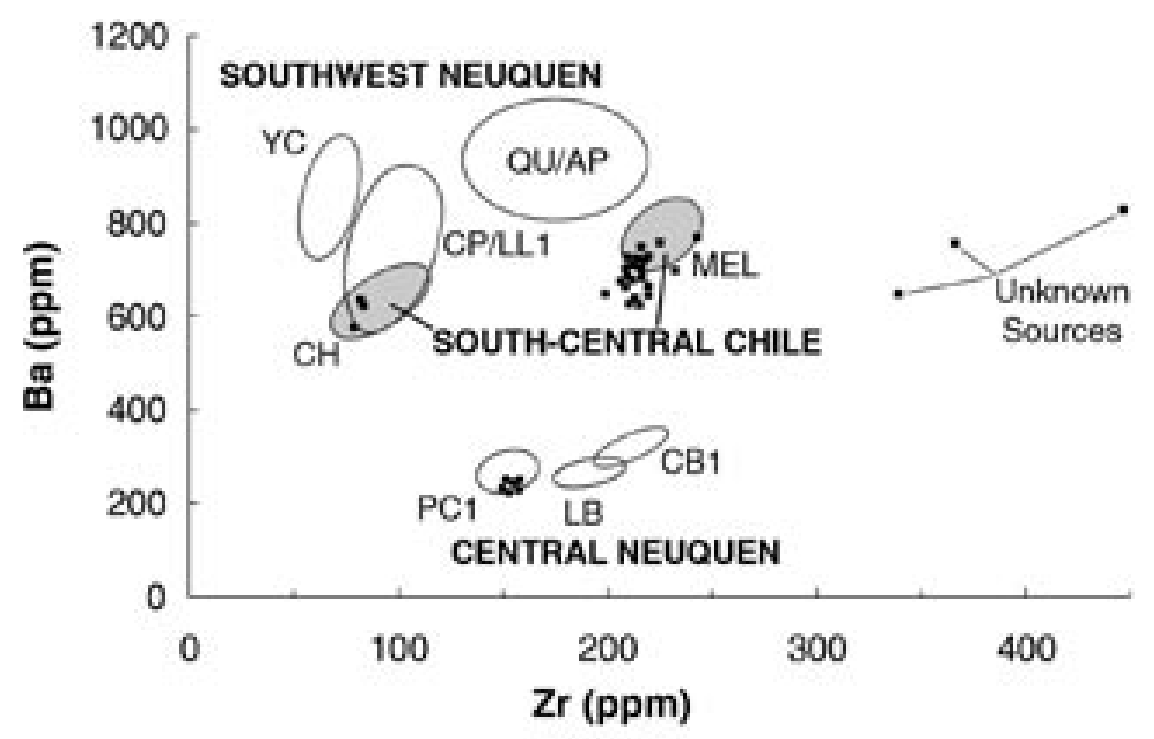

Fig. 3. Ba versus Zr para muestras de sitios arqueológicos del centro-sur de en Chile centro-sur (cuadrados pequeños; Tabla 2) comparado con las composiciones de obsidianas de fuentes conocidas en la región, incluye ambas, MEL de los Nevados de Sollipulli (Stern et al., 2008) y CH el volcán Chaitén (Stern et al., 2002) en Chile centro-sur (campos sombreados); YC, QU/ AP y CP/LL1 de las zonas de Lagos Lacar y Lolog en el suroeste de Neuquén, Argentina (López et al., 2009); y PC1, LB y CB1 de Portada Covunco, Cerro Bajo y La Bandera (Bellelli et al., 2002 y 2006; López et al., 2009). Tres de las muestras de obsidianas negras analizadas son de composición diferente a la de otras obsidianas de fuentes conocidas. Estas tres muestras de obsidiana negra tienen altas concentraciones de Zry de otros elementos de alto potencial iónico (HFSE) y tierras raras. Todas las otras obsidianas negras son similares a la fuente MEL de los Nevados de Sollipulli. Tres muestras de obsidiana gris de Chanchán son similares a la obsidiana $\mathrm{CH}$ del volcán Chaitén. Cinco muestras de obsidianas translucidas y cuatro muestras de obsidianas rojas son similares a la obsidiana PC1 de Portada Covunco en Neuquén central al este del Paso de Icalma (Fig. 1). 
Tabla 2. Elementos en trazas (ppm) en obsidianas negras del sitio Alero Cabeza de Indio-1.

\begin{tabular}{|c|c|c|c|c|c|c|c|c|c|}
\hline muestra & CS351 & CS352 & CS352-AD & CS353 & CS354 & CS354 new & CS355 & CS356 & CS357 \\
\hline Tipo & MEL1 & MEL1 & MEL1 & MEL1 & MEL1 & MEL1 & MEL1 & MEL1 & MEL1 \\
\hline & & & & & & & & & \\
\hline $\mathrm{Ti}^{*}$ & 1363 & 1391 & 1346 & 1301 & 1338 & 1358 & 1345 & 1350 & 1317 \\
\hline $\mathrm{Mn}$ & 388 & 395 & 371 & 392 & 387 & 429 & 388 & 416 & 410 \\
\hline & & & & & & & & & \\
\hline $\mathrm{Cs}$ & 4,8 & 5,0 & 4,8 & 4,8 & 4,9 & 4,6 & 4,8 & 4,8 & 5,0 \\
\hline $\mathrm{Rb}$ & 109 & 112 & 105 & 111 & 110 & 99 & 109 & 110 & 114 \\
\hline $\mathrm{Sr}$ & 125 & 128 & 125 & 120 & 127 & 122 & 129 & 121 & 123 \\
\hline $\mathrm{Ba}$ & 727 & 747 & 707 & 637 & 698 & 626 & 719 & 628 & 651 \\
\hline & & & & & & & & & \\
\hline $\mathrm{Y}$ & 14 & 15 & 14 & 14 & 15 & 15 & 14 & 14 & 15 \\
\hline $\mathrm{Zr}$ & 210 & 217 & 208 & 214 & 214 & 211 & 217 & 216 & 219 \\
\hline $\mathrm{Nb}$ & 7,3 & 7,4 & 7,4 & 6,7 & 6,8 & 6,2 & 6,7 & 7,3 & 6,4 \\
\hline $\mathrm{Hf}$ & 5,3 & 5,5 & 5,1 & 5,3 & 5,3 & 5,3 & 5,3 & 5,6 & 5,5 \\
\hline $\mathrm{Pb}$ & 25,2 & 23,9 & 22,7 & 22,9 & 22,6 & 22,2 & 22,9 & 24,7 & 23,8 \\
\hline $\mathrm{Th}$ & 11,1 & 11,4 & 10,9 & 11,1 & 10,8 & 8,7 & 11,1 & 11,8 & 11,4 \\
\hline $\mathrm{U}$ & 2,8 & 2,9 & 2,7 & 2,7 & 2,8 & 2,6 & 2,8 & 3,0 & 2,9 \\
\hline & & & & & & & & & \\
\hline $\mathrm{La}$ & 20,9 & 21,2 & 20,2 & 18,5 & 20,3 & 21,2 & 20,8 & 17,6 & 19,1 \\
\hline $\mathrm{Ce}$ & 39,2 & 39,9 & 37,9 & 35,4 & 38,7 & 41,5 & 38,8 & 34,5 & 36,2 \\
\hline $\mathrm{Pr}$ & 4,24 & 4,17 & 3,94 & 3,73 & 4,11 & 4,26 & 4,14 & 3,85 & 3,87 \\
\hline $\mathrm{Nd}$ & 15,5 & 15,6 & 14,7 & 15,0 & 15,4 & 17,3 & 15,6 & 15,8 & 15,6 \\
\hline $\mathrm{Sm}$ & 2,73 & 2,64 & 2,66 & 2,75 & 2,88 & 3,07 & 2,66 & 2,79 & 2,70 \\
\hline $\mathrm{Eu}$ & 0,64 & 0,68 & 0,66 & 0,66 & 0,70 & 0,84 & 0,68 & 0,73 & 0,74 \\
\hline $\mathrm{Gd}$ & 2,88 & 2,94 & 2,81 & 2,77 & 2,92 & 2,95 & 2,83 & 2,67 & 2,89 \\
\hline $\mathrm{Tb}$ & 0,39 & 0,34 & 0,36 & 0,34 & 0,35 & 0,38 & 0,37 & 0,33 & 0,31 \\
\hline $\mathrm{Dy}$ & 2,31 & 2,33 & 2,41 & 2,15 & 2,33 & 2,47 & 2,23 & 2,23 & 2,26 \\
\hline $\mathrm{Ho}$ & 0,48 & 0,50 & 0,46 & 0,43 & 0,49 & 0,49 & 0,49 & 0,46 & 0,44 \\
\hline $\mathrm{Er}$ & 1,57 & 1,64 & 1,59 & 1,57 & 1,60 & 1,64 & 1,69 & 1,55 & 1,65 \\
\hline $\mathrm{Tm}$ & 0,22 & 0,21 & 0,24 & 0,22 & 0,22 & 0,21 & 0,24 & 0,20 & 0,21 \\
\hline $\mathrm{Yb}$ & 1,81 & 1,79 & 1,73 & 1,64 & 1,64 & 1,98 & 1,76 & 1,80 & 1,83 \\
\hline $\mathrm{Lu}$ & 0,25 & 0,26 & 0,25 & 0,24 & 0,25 & 0,26 & 0,28 & 0,25 & 0,26 \\
\hline
\end{tabular}

centraciones más bajas de Sr y Ba, además de concentraciones mayores de todos los otros elementos trazas, y se considera como una obsidiana (ACI2) relacionada a ACI1.

La obsidiana tipo ACI1 es químicamente similar a la obsidiana PC1 encontrada al este del drenaje de la Cordillera Andina en Portada Covunco en Neuquén Central, Argentina (Fig. 1). Estas muestras son similares en contenido de Ba (entre 250-300 ppm, Fig. 3) a varias otras obsidianas de Neuquén Central, pero tienen un contenido de $\operatorname{Zr}(\sim 150 \mathrm{ppm})$ relativamente bajo, semejante a las muestras de Portada Covunco PC1 (Fig. 3).

\section{DISCUSIÓN Y CONCLUSIONES}

La presencia de la obsidiana negra tipo MEL en los sitios terrestres del centro-sur de Chile índica una dispersión substancial de este tipo de obsidiana, realizada por cazadores-recolectores a lo largo de la Depresión Central y a lo ancho de la Cordillera Andina Principlal. La presencia de este mismo tipo de obsidiana negra en Chan Chan-18, donde esta obsidiana y la obsidiana del Volcán Chaitén coexisten, sugiere la posibilidad de intercambio de material entre cazadores-recolectores terrestres y grupos canoeros. Esta posibilidad es confirmada por la dispersión de la obsidiana negra tipo MEL, producto de la movilidad de los canoeros, hasta 
Tabla 3. Elementos en trazas (ppm) en obsidianas translucidas y rojas de sitios arqueológicos del centro-sur de Chile.

\begin{tabular}{|c|c|c|c|c|c|c|c|c|c|c|}
\hline sitio & \multicolumn{5}{|c|}{ Alero Cabeza de Indio 1} & \multicolumn{4}{|c|}{ Alero Cabeza de Indio 1} & Flor del Lago 1 \\
\hline lab \# & CS358 & CS359 & CS359 new & CS360 & CS361 & CS362 & CS363 & CS364 & CS365 & CS376 \\
\hline color & traslúcida & traslúcida & traslúcida & traslúcida & traslúcida & rojo & rojo & rojo & rojo & traslúcida \\
\hline tipo & ACI1 & $\mathrm{ACI} 2$ & $\mathrm{ACI} 2$ & ACI1 & ACI1 & ACI1 & ACI1 & ACI1 & ACI1 & ACI1 \\
\hline $\mathrm{Ti}^{*}$ & 1030 & 987 & 998 & 979 & 1022 & 1022 & 966 & 976 & 931 & 1045 \\
\hline $\mathrm{Mn}$ & 404 & 440 & 445 & 388 & 421 & 420 & 398 & 399 & 405 & 408 \\
\hline $\mathrm{Cs}_{\mathrm{s}}$ & 6.9 & 9.5 & 96 & 67 & 67 & 6.8 & 67 & 69 & 67 & 6.9 \\
\hline $\mathrm{Rb}$ & 162 & 193 & 190 & 159 & 161 & 164 & 160 & 165 & 161 & 163 \\
\hline $\mathrm{Sr}$ & 49 & 10 & 10 & 47 & 49 & 49 & 44 & 47 & 47 & 49 \\
\hline $\mathrm{Ba}$ & 244 & 26 & 28 & 237 & 250 & 244 & 225 & 233 & 244 & 254 \\
\hline $\mathrm{Y}$ & 17 & 19 & 18 & 16 & 17 & 17 & 17 & 17 & 16 & 17 \\
\hline $\mathrm{Zr}$ & 155 & 164 & 165 & 150 & 153 & 156 & 153 & 156 & 158 & 159 \\
\hline $\mathrm{Nb}$ & 28,0 & 35,1 & 36,0 & 27,1 & 27,8 & 29,1 & 27,9 & 28,5 & 29,0 & 30,4 \\
\hline $\mathrm{Hf}$ & 5,1 & 5,5 & 5,7 & 5,0 & 5,1 & 5,3 & 5,0 & 5,1 & 5,9 & 5,0 \\
\hline $\mathrm{Pb}$ & 19,8 & 20,7 & 20,6 & 18,9 & 20,0 & 19,5 & 20,2 & 20,1 & 19,1 & 20,8 \\
\hline Th & 25,8 & 28,3 & 28,9 & 24,6 & 25,7 & 26,1 & 25,7 & 25,5 & 26,5 & 26,5 \\
\hline $\mathrm{U}$ & 6,6 & 7,5 & 7,7 & 6,3 & 6,6 & 6,7 & 6,5 & 6,6 & 6,7 & 6,9 \\
\hline $\mathrm{La}$ & 30,4 & 35,3 & 35,1 & 29,7 & 31,0 & 30,5 & 27,8 & 29,0 & 30,0 & 31,0 \\
\hline $\mathrm{Ce}$ & 54,9 & 64,6 & 63,9 & 52,9 & 55,1 & 55,9 & 51,3 & 53,4 & 55,0 & 56,2 \\
\hline $\operatorname{Pr}$ & 5,59 & 6,62 & 6,58 & 5,35 & 5,56 & 5,75 & 5,19 & 5,27 & 5,60 & 5,59 \\
\hline $\mathrm{Nd}$ & 19,1 & 22,3 & 22,3 & 18,8 & 18,7 & 18,7 & 18,5 & 18,9 & 17,9 & 20,7 \\
\hline $\mathrm{Sm}$ & 3,10 & 3,38 & 3,35 & 3,04 & 3,02 & 3,00 & 2,95 & 2,95 & 3,08 & 3,19 \\
\hline $\mathrm{Eu}$ & 0,36 & 0,10 & 0,10 & 0,38 & 0,42 & 0,43 & 0,40 & 0,39 & 0,46 & 0,38 \\
\hline Gd & 3,29 & 4,12 & 4,15 & 3,17 & 3,76 & 3,55 & 3,41 & 3,21 & 3,33 & 3,38 \\
\hline $\mathrm{Tb}$ & 0,44 & 0,53 & 0,54 & 0,39 & 0,45 & 0,43 & 0,39 & 0,38 & 0,48 & 0,42 \\
\hline Dy & 2,81 & 3,18 & 3,15 & 2,60 & 2,68 & 2,81 & 2,79 & 2,72 & 2,82 & 2,88 \\
\hline $\mathrm{Ho}$ & 0,53 & 0,64 & 0,62 & 0,55 & 0,58 & 0,50 & 0,51 & 0,49 & 0,65 & 0,58 \\
\hline $\mathrm{Er}$ & 1,84 & 2,23 & 2,21 & 1,87 & 1,80 & 1,83 & 1,80 & 1,74 & 1,88 & 1,88 \\
\hline $\mathrm{Tm}$ & 0,25 & 0,28 & 0,27 & 0,27 & 0,24 & 0,27 & 0,26 & 0,26 & 0,31 & 0,28 \\
\hline $\mathrm{Yb}$ & 2,09 & 2,32 & 2,35 & 2,00 & 2,25 & 2,13 & 2,10 & 2,09 & 2,20 & 2,27 \\
\hline $\mathrm{Lu}$ & 0,29 & 0,33 & 0,32 & 0,32 & 0,30 & 0,33 & 0,29 & 0,30 & 0,34 & 0,31 \\
\hline
\end{tabular}

tan al sur como Puente Quilo, en la Isla Grande de Chiloé.

Los datos sugieren que la obsidiana tipo PC1 encontrada en Portada Covunco, en Argentina, cruzó la divisoria de aguas entre Chile y Argentina, posiblemente a través del Paso de Icalma, el paso de menor altura en la zona. Alternativamente, esta obsidiana podría tener otra fuente primaria aun no identificada a lo largo de la cadena volcánica que forma la divisoria continental al norte de este paso. La fuente secundaria de la obsidiana tipo PC1 en el drenaje fluvial en Portada Covunco consiste de bochones redondeados, los que deben tener una fuente primaria más al oeste en la cadena de volcanes y calderas que corre desde el Volcán Copahue, por el norte, hacia el sureste a través de Pino Hachado, de la caldera de Palo Mahuida y del cono de Queli Mahuida, inmediatamente al norte del Paso de Icalma (Fig. 1, Muñoz y Stern, 1989). Esta cadena ha producido grandes volúmenes de rocas piroclásticas riolíticas y es probable que contenga domos de obsidiana riolítica, aún no reconocidos, los que podrían ser la fuente primaria de la obsidiana PC1 encontrada en la Portada Covunco.

Aunque la fuente de Portada Covunco no se encuentra muy distante del sitio Alero Cabeza de Indio-1, $\approx 70 \mathrm{~km}$ al este, igualmente está más lejos de este sitio que la fuente de la obsidiana negra MEL 


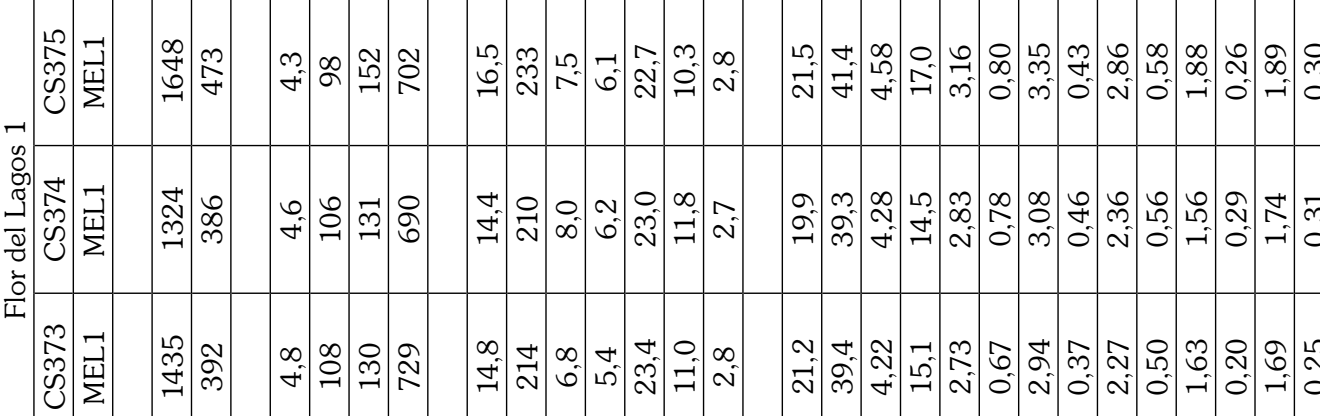

\begin{tabular}{|c|c|c|c|c|c|c|c|c|c|c|c|c|c|c|c|c|c|c|c|c|c|c|c|c|c|c|c|c|}
\hline $\begin{array}{l}\overrightarrow{1} \\
\tilde{n} \\
0\end{array}$ & $\begin{array}{l}N \\
\tilde{N} \\
\mathcal{U}\end{array}$ & 立 & $\begin{array}{l}\stackrel{2}{\Omega} \\
\stackrel{m}{n}\end{array}$ & $\frac{a}{n}$ & $\stackrel{\infty}{\infty}$ & : & $\widehat{\cong}$ & $\underset{\nabla}{\Delta}$ & $\underset{\Xi}{\Delta}$ & 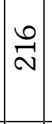 & a. & $\begin{array}{l}0 \\
0\end{array}$ & $\begin{array}{l}0 \\
\text { Nै}\end{array}$ & \begin{tabular}{c|c}
$\infty$ \\
0 \\
0
\end{tabular} & 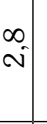 & $\begin{array}{l}0 \\
2 \\
\sim\end{array}$ & $\begin{array}{l}\Omega \\
\tilde{n}\end{array}$ & $\begin{array}{ll}0 \\
0 \\
+i\end{array}$ & $\begin{array}{l}+ \\
\dot{1} \\
\dot{1}\end{array}$ & 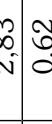 & $\begin{array}{c}v \\
0 \\
0 \\
0\end{array}$ & 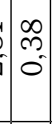 & $\begin{array}{l}\stackrel{L}{N} \\
\text { N }\end{array}$ & 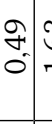 & 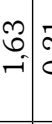 & 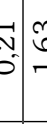 & & 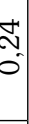 \\
\hline 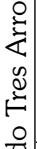 & $\begin{array}{l}\mathcal{N} \\
\tilde{n} \\
\tilde{U}\end{array}$ & 点 & 品 & 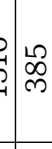 & 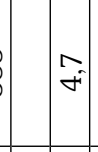 & $\mid \begin{array}{l}\infty \\
0 \\
-1\end{array}$ & 工ี & $\widehat{\widehat{\sigma}}$ & $\underset{\Xi}{む}$ & $\stackrel{\sim}{\stackrel{N}{N}}$ & $\mid$ & $\begin{array}{l}m \\
\omega\end{array}$ & 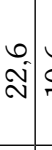 & \begin{tabular}{l|c}
0 \\
0 \\
0 & 0
\end{tabular} & 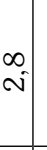 & बे & $\begin{array}{l}5 \\
\hat{n}\end{array}$ & \begin{tabular}{lll}
8 & \multicolumn{1}{c}{} \\
0 & 5
\end{tabular} & $\begin{array}{c}0 \\
10 \\
-1\end{array}$ & \begin{tabular}{l|l}
0 & \\
$\infty$ & \\
$-v$ & 0
\end{tabular} & 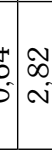 & $\begin{array}{l}\hat{n} \\
\tilde{j} \\
\tilde{j} \\
\tilde{0}\end{array}$ & $\begin{array}{l}\hat{N} \\
\text { N }\end{array}$ & $\begin{array}{lll}0 & 0 \\
+ \\
0 & \\
0\end{array}$ & \begin{tabular}{l|c}
$\infty$ & \\
\llcorner & \\
-1 &
\end{tabular} & \begin{tabular}{l|l}
$N^{N}$ & $\bar{a}$ \\
$b^{-}$ &
\end{tabular} & 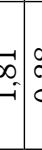 & $\begin{array}{l}\infty \\
\text { N } \\
0 \\
0\end{array}$ \\
\hline$\underset{\mid}{3}$ & $\mid \begin{array}{l}\stackrel{0}{N} \\
\tilde{n} \\
\mathcal{U}\end{array}$ & 点 & $\begin{array}{l}\mathcal{N} \\
\tilde{w} \\
-1\end{array}$ & 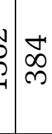 & $\begin{array}{l}\infty \\
+ \\
+\end{array}$ & 。 & $\stackrel{\substack{\infty \\
\sim}}{\sim}$ & $\stackrel{\curvearrowright}{=}$ & $\vec{\Xi}$ & $\stackrel{m}{\sim}$ & $\begin{array}{l}0 \\
6\end{array}$ & $\begin{array}{l}N \\
\tilde{N}^{\circ}\end{array}$ & ָิ & \begin{tabular}{l|l}
0 & 0 \\
& 0
\end{tabular} & $\begin{array}{l}\infty \\
0 \\
v\end{array}$ & 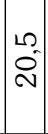 & $\begin{array}{c}m \\
\infty \\
m\end{array}$ & 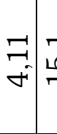 & حิ & \begin{tabular}{c|c}
$v$ & 0 \\
$v$ & 0 \\
$v$ &
\end{tabular} & \begin{tabular}{l|l}
0 \\
0 \\
\hdashline & $\infty$ \\
\hdashline & $\infty$ \\
\end{tabular} & $\begin{array}{l}\tilde{n} \\
\tilde{j} \\
\tilde{0}\end{array}$ & \begin{tabular}{l} 
Oे \\
\multirow{2}{*}{} \\
vis
\end{tabular} & $\begin{array}{ccc}0 & \\
+ \\
0 \\
0\end{array}$ & $\begin{array}{l}\text { ⿵్ర } \\
-\end{array}$ & \begin{tabular}{c|c}
$N$ & 7 \\
5 & 7
\end{tabular} & & $\begin{array}{l}\stackrel{d}{N} \\
\text { O. }\end{array}$ \\
\hline & $\begin{array}{l}0 \\
0 \\
\tilde{n} \\
\tilde{\omega}\end{array}$ & 点 & $\begin{array}{l}\stackrel{\omega}{m} \\
m \\
\sim\end{array}$ & 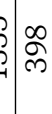 & g & 元 & 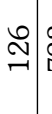 & 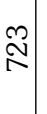 & $\underset{\Xi}{ \pm}$ & $\stackrel{\infty}{\underset{\sim}{\sim}}$ & $\begin{array}{c}5 \\
6\end{array}$ & $\begin{array}{c}4 \\
10\end{array}$ & $\begin{array}{l}m \\
\tilde{N} \\
\sim\end{array}$ & $\begin{array}{l}0 \\
0 \\
0\end{array}$ & $\begin{array}{l}\infty \\
\sim \\
\sim\end{array}$ & $\begin{array}{l}0 \\
0 \\
0 \\
N\end{array} \mid$ & $\begin{array}{c}\vec{\infty} \\
m \\
m\end{array}$ & $\begin{array}{ll}9 \\
0 \\
\forall\end{array}$ & مْ & $\begin{array}{c}-v \\
\frac{2}{2}\end{array}$ & \begin{tabular}{lll}
0 & 0 \\
\hdashline & $\infty$ \\
\hdashline
\end{tabular} & $\begin{array}{ll}0 \\
j \\
j \\
0 \\
0\end{array}$ & $\begin{array}{l}\text { D. } \\
\text { vi }\end{array}$ & $\begin{array}{lll}\infty & \\
+ \\
0\end{array}$ & అ్రి| & 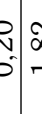 & & 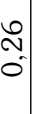 \\
\hline $\begin{array}{l}\overrightarrow{1} \\
0 \\
0 \\
0 \\
0 \\
\alpha\end{array}$ & $\begin{array}{l}\infty \\
0 \\
\tilde{n} \\
\tilde{\omega}\end{array}$ & 立 & 守 & $\begin{array}{l}+ \\
+ \\
+\end{array}$ & 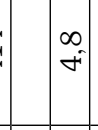 & 윽 & $\stackrel{\llcorner}{\sim}$ & స్రి & 늠 & $\stackrel{\infty}{\sim}$ & $\begin{array}{l}\infty \\
6\end{array}$ & $\begin{array}{l}0 \\
0\end{array}$ & $\begin{array}{l}\Omega \\
\text { Nै }\end{array}$ & \begin{tabular}{l|l}
0 & 0 \\
-1 & 0
\end{tabular} & $\begin{array}{l}\infty \\
\cdots \\
\sim\end{array}$ & $\begin{array}{c}0 \\
\infty \\
-1\end{array}$ & $\begin{array}{l}0 \\
0 \\
\infty\end{array} \mid$ & $\begin{array}{l}\text { ఇ. } \\
\text { m. }\end{array}$ & 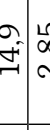 & \begin{tabular}{c|c}
$\infty$ & $\Re$ \\
$i v$ & 0
\end{tabular} & 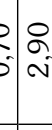 & $\hat{j}$ & \begin{tabular}{c}
0 \\
\multicolumn{1}{c}{} \\
v
\end{tabular} & 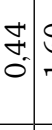 & రి & 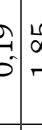 & శ) & $\begin{array}{c}0 \\
\text { N. } \\
0 \\
0\end{array}$ \\
\hline $\begin{array}{l}1 \\
0 \\
0 \\
.1\end{array}$ & $\begin{array}{l}\hat{\sigma} \\
\tilde{\omega} \\
\tilde{U}\end{array}$ & 㤩 & $\begin{array}{l}\overline{6} \\
\text { m }\end{array}$ & 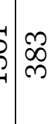 & $\begin{array}{l}\infty \\
f^{\infty}\end{array}$ & $\stackrel{2}{0}$ & શิ) & ๖ำ & 志 & $\stackrel{\sim}{\sim}$ & $\begin{array}{l}0 \\
6\end{array}$ & $\begin{array}{l}N \\
\sim \\
\sim\end{array}$ & $\begin{array}{l}\infty \\
\stackrel{N}{N}\end{array}$ & : & $\widehat{v}$ & 겅 & $\mid \begin{array}{l}10 \\
\infty \\
\infty \\
m\end{array}$ & $\begin{array}{ll}5 \\
0 \\
\sigma^{\prime}\end{array}$ & \begin{tabular}{l|l}
0 \\
$\stackrel{0}{0}$ \\
-1
\end{tabular} & $\begin{array}{ll}v & n \\
v & 0\end{array}$ & $\begin{array}{lll}0 \\
0 \\
0\end{array}$ & $\begin{array}{c}m \\
m \\
0 \\
0\end{array}$ & $\vec{m}$ & $\begin{array}{ll}\dot{H} \\
0\end{array}$ & \begin{tabular}{l|l}
0 & \\
+ & \\
- &
\end{tabular} & $\begin{array}{ll}\mathbf{N} & 7 \\
0^{-} & \end{array}$ & 6 & $\begin{array}{l}\vec{N} \\
0 \\
0\end{array}$ \\
\hline & $\begin{array}{l}0 \\
0 \\
\tilde{D} \\
\tilde{U}\end{array} \mid$ & 实 & 苞 & 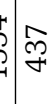 & $\begin{array}{l}0 \\
+\end{array}$ & $\begin{array}{l}m \\
0 \\
-1\end{array}$ & ন & فํ. & 志 & $\underset{\Im}{\stackrel{\sim}{\sim}}$ & $\begin{array}{l}a \\
\infty\end{array}$ & $\stackrel{m}{\sim}$ & డీ & \begin{tabular}{l|l}
$\infty$ & 0 \\
$=$ & 0
\end{tabular} & 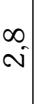 & $\begin{array}{l}\Omega \\
\widehat{N}\end{array}$ & $\begin{array}{l}0 \\
0 \\
10\end{array}$ & \begin{tabular}{c|c}
$\infty$ & \\
+ \\
6
\end{tabular} & 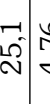 & $\begin{array}{cc}0 & 0 \\
+ & \sigma\end{array}$ & 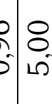 & $\begin{array}{ll}0 & 0 \\
0 & 0 \\
0\end{array}$ & $\begin{array}{l}5 \\
0\end{array}$ & $\begin{array}{ll}+ & \\
\infty & \\
0 & 0\end{array}$ & 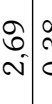 & \begin{tabular}{c|c}
0 \\
$m$ \\
$b^{0}$
\end{tabular} & $\begin{array}{c}8 \\
ن \\
ن\end{array}$ & $\begin{array}{l}-7 \\
0 \\
0\end{array}$ \\
\hline $\begin{array}{c}N \\
0 \\
0 \\
0 \\
\mathbb{0} \\
0 \\
0 \\
0 \\
0\end{array}$ & $\left|\begin{array}{l}m \\
* \\
\vdots \\
0 \\
\forall\end{array}\right|$ & ๙:: & $\begin{array}{l}2 \\
1 \\
1\end{array}$ & 太 & $\sigma^{6}$ & \begin{tabular}{c}
$N$ \\
\\
\hdashline
\end{tabular} & m) & 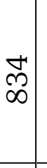 & ชิ & $\underset{g}{g}$ & $\begin{array}{l}a \\
\sigma\end{array}$ & $\begin{array}{l}a \\
0 \\
0\end{array}$ & $\begin{array}{l}0 \\
\text { mit }\end{array}$ & $\begin{array}{l}0 \\
\text { m. }\end{array}$ & $\vec{m}$ & $\begin{array}{c}\hat{m} \\
\tilde{m}\end{array}$ & $\mid \begin{array}{c}0 \\
\infty \\
\infty\end{array}$ & $\begin{array}{l}\text { అิ| } \\
0 \\
0\end{array}$ & $\begin{array}{ll}m & 4 \\
g & \end{array}$ & 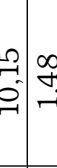 & 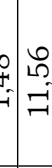 & \begin{tabular}{l}
$R$ \\
\hdashline \\
-1
\end{tabular} & $\begin{array}{l}0 \\
0 \\
0 \\
0\end{array}$ & $\begin{array}{ll}N & \\
N & \\
N\end{array}$ & \begin{tabular}{l|l}
0 \\
6 \\
6
\end{tabular} & & ton & $\begin{array}{l}0 \\
0 \\
-i\end{array}$ \\
\hline 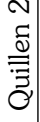 & $\begin{array}{l}N \\
¥ \\
0 \\
0 \\
\forall\end{array} \mid$ & ๙: & $\underset{\sim}{\stackrel{Z}{N}}$ & 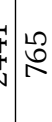 & 6 & 吉 & చొ & 금 & Y & $\hat{\tilde{b}}$ & $\begin{array}{l}N \\
a\end{array}$ & $\begin{array}{c}\infty \\
\stackrel{-}{\sim} \\
\sim\end{array}$ & 号 & $\begin{array}{l}\text { N } \\
\stackrel{0}{0} \\
\text { ஸी }\end{array}$ & $\overrightarrow{\dot{m}}$ & $\begin{array}{l}\text { ฮे } \\
\text { సे }\end{array}$ & $\left|\begin{array}{c}\infty \\
\infty \\
0 \\
0\end{array}\right|$ & 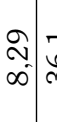 & 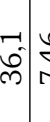 & \begin{tabular}{l}
0 \\
\multirow{2}{*}{} \\
\end{tabular} & $\begin{array}{ll}0 & \curvearrowright \\
+ & \infty \\
-1 & \infty\end{array}$ & $\hat{v}$ & 이. & g & & & $\frac{N}{f}$ & $\begin{array}{l}-1 \\
0 \\
0\end{array}$ \\
\hline 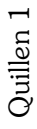 & 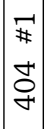 & $\stackrel{\overrightarrow{1}}{\mathbf{s}}$ & $\begin{array}{l}0 \\
\infty \\
10 \\
\end{array}$ & $\begin{array}{c}b_{-1}^{0} \\
\end{array}$ & $\begin{array}{c}0 \\
10 \\
10\end{array}$ & 늑 & ભి & 灾 & 늠 & $\stackrel{L}{\sim}$ & $\begin{array}{l}n \\
6\end{array}$ & \begin{tabular}{l|l}
$\infty$ & \\
0 \\
0
\end{tabular} & $\begin{array}{l}m \\
\dot{0}\end{array}$ & \begin{tabular}{l}
$\Omega$ \\
\multirow{N}{*}{}
\end{tabular} & $\begin{array}{l}N \\
m^{-1}\end{array}$ & $\begin{array}{l}10 \\
0 \\
\mathcal{N}\end{array}$ & $\begin{array}{l}\infty \\
\rho \\
\infty\end{array}$ & $\begin{array}{lll}\infty & 0 \\
7 & 5\end{array}$ & 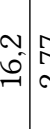 & \begin{tabular}{c|c}
$\hat{N}$ & $\infty$ \\
0
\end{tabular} & \begin{tabular}{c|c}
0 \\
0 \\
0 \\
\hdashline & $\infty$ \\
$\sim$
\end{tabular} & 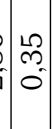 & \begin{tabular}{c}
2 \\
\multirow{v}{v}{}
\end{tabular} & $\begin{array}{ll}0 & \\
+ \\
0\end{array}$ & $\begin{array}{l}g \\
-i\end{array}$ & & $\stackrel{0}{2}$ & $\begin{array}{l}\stackrel{\sim}{N} \\
\stackrel{0}{0}\end{array}$ \\
\hline$\frac{9}{\omega}$ & $\begin{array}{l}* \\
0 \\
0 \\
0\end{array}$ & | & $\stackrel{*}{i}$ & $=\sum^{\mathcal{L}}$ & $3^{3}$ & $\overrightarrow{\hat{x}}$ & $\grave{\omega}$ & $\tilde{\varpi}$ & $>$ & 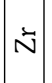 & Z & $F$ & 岌 & $\overrightarrow{0}=$ & $\supset$ & $\stackrel{\widetilde{\sigma}}{\lrcorner}$ & $U^{0}$ & $\Sigma^{2}$ & $\bar{z}$ & ตี & $\begin{array}{l}7 \\
\end{array}$ & $\hat{F}$ & $a^{n}=$ & $\stackrel{\text { : }}{\text { I }}$ & जี & $\xi$ & خ & |בَ \\
\hline
\end{tabular}


Tabla 5. Elementos en trazas (ppm) en obsidianas negras y grises de sitios costeros de Chan Chan-18 y Puente Quilo.

\begin{tabular}{|c|c|c|c|c|c|c|c|c|c|}
\hline lab \# & $407 \# 4$ & $408 \# 5$ & $409 \# 6$ & $410 \# 7$ & $411 \# 8$ & $412 \# 9$ & 401 & $402 \# 040$ & $403 \# 039$ \\
\hline sitio & Chan Chan & Chan Chan & Chan Chan & Chan Chan & Chan Chan & Chan Chan & Quilo & Quilo & Quilo \\
\hline color & negra & gris & negra & negra & gris & gris & negra & negra & negra \\
\hline tipo & desconocido & $\mathrm{CH}$ & MEL1 & MEL1 & $\mathrm{CH}$ & $\mathrm{CH}$ & MEL1 & MEL1 & MEL1 \\
\hline $\mathrm{Ti}^{*}$ & 1989 & 1080 & 1786 & 1658 & 969 & 993 & 1843 & 1810 & 1805 \\
\hline $\mathrm{Mn}$ & 700 & 560 & 459 & 429 & 539 & 568 & 402 & 412 & 392 \\
\hline Cs & 5,8 & 7.4 & 4.9 & 4.6 & 7.2 & 7.5 & 4.7 & 5,0 & 49 \\
\hline $\mathrm{Rb}$ & 92 & 127 & 110 & 99 & 120 & 125 & 104 & 110 & 109 \\
\hline $\mathrm{Sr}$ & 116 & 154 & 140 & 122 & 142 & 153 & 114 & 116 & 115 \\
\hline $\mathrm{Ba}$ & 654 & 634 & 757 & 626 & 587 & 645 & 648 & 664 & 673 \\
\hline Y & 47 & 12 & 16 & 15 & 11 & 12 & 14 & 14 & 14 \\
\hline $\mathrm{Zr}$ & 340 & 83 & 225 & 211 & 79 & 82 & 198 & 208 & 207 \\
\hline $\mathrm{Nb}$ & 8,2 & 10,6 & 8,0 & 6,2 & 8,9 & 9,6 & 5,8 & 6,5 & 6,0 \\
\hline Th & 7,2 & 13,6 & 10,5 & 8,7 & 11,8 & 13,1 & 9,0 & 10,1 & 9,3 \\
\hline $\mathrm{Hf}$ & 9,4 & 3,5 & 5,5 & 5,3 & 2,6 & 2,9 & 5,1 & 6,4 & 5,2 \\
\hline $\mathrm{Pb}$ & 25,6 & 36,3 & 26,9 & 22,2 & 19,7 & 27,2 & 21,8 & 23,5 & 22,5 \\
\hline $\mathrm{U}$ & 2,2 & 3,7 & 3,1 & 2,6 & 3,4 & 3,7 & 2,7 & 2,9 & 2,8 \\
\hline $\mathrm{La}$ & 28,3 & 25,8 & 21,2 & 18,2 & 24,3 & 25,6 & 18,0 & 18,8 & 18,5 \\
\hline $\mathrm{Ce}$ & 54,9 & 49,8 & 41,5 & 36,4 & 47,5 & 50,1 & 35,1 & 36,2 & 35,2 \\
\hline $\mathrm{Pr}$ & 7,08 & 5,11 & 4,26 & 3,72 & 4,71 & 5,10 & 3,61 & 3,82 & 3,70 \\
\hline $\mathrm{Nd}$ & 32,4 & 19,1 & 17,3 & 15,5 & 18,6 & 18,5 & 15,9 & 16,2 & 14,8 \\
\hline $\mathrm{Sm}$ & 7,3 & 3,1 & 3,1 & 2,9 & 3,0 & 3,0 & 2,6 & 2,8 & 2,6 \\
\hline $\mathrm{Eu}$ & 1,4 & 0,7 & 0,8 & 0,8 & 0,6 & 0,7 & 0,6 & 0,7 & 0,7 \\
\hline $\mathrm{Gd}$ & 8,5 & 3,1 & 3,0 & 3,1 & 2,9 & 2,9 & 2,9 & 3,0 & 2,7 \\
\hline $\mathrm{Tb}$ & 1,1 & 0,3 & 0,4 & 0,4 & 0,3 & 0,3 & 0,3 & 0,3 & 0,3 \\
\hline Dy & 8,0 & 2,0 & 2,5 & 2,3 & 1,9 & 2,1 & 2,3 & 2,3 & 2,2 \\
\hline Ho & 1,6 & 0,4 & 0,5 & 0,5 & 0,3 & 0,4 & 0,4 & 0,4 & 0,4 \\
\hline $\mathrm{Er}$ & 5,2 & 1,2 & 1,6 & 1,6 & 1,2 & 1,2 & 1,4 & 1,4 & 1,5 \\
\hline $\mathrm{Tm}$ & 0,7 & 0,1 & 0,2 & 0,2 & 0,1 & 0,1 & 0,2 & 0,2 & 0,2 \\
\hline $\mathrm{Yb}$ & 5,5 & 1,2 & 2,0 & 1,8 & 1,3 & 1,3 & 1,8 & 1,8 & 1,7 \\
\hline $\mathrm{Lu}$ & 0,8 & 0,2 & 0,3 & 0,3 & 0,2 & 0,2 & 0,2 & 0,2 & 0,2 \\
\hline
\end{tabular}

proveniente desde solo $30 \mathrm{~km}$ al sur en los Nevados de Sollipulli. Esto explica la mayor abundancia de la obsidiana MEL en este sitio comparado con la obsidiana PC1.

Las muestras de obsidiana tipo PC1 en el centro-sur de Chile son todas desde translucidas a transparentes o rojas y visualmente distintivas de la obsidiana negra MEL. Sin embargo, la fuente de la obsidiana de PC1 en la Portada Covunco también incluye tipos de coloración negro oscuro. El hecho que todas las muestras de tipo PC1 en los sitios chilenos sean visualmente distintivas de la obsidiana negra MEL sugiere de que las obsidianas translucidas y rojas podrían haber tenido un valor estético mayor.
Alternativamente, estudios detallados de los sitios chilenos podrían permitir descubrir obsidianas negras provenientes desde esa fuente más distante.

Finalmente, de las 35 muestras de obsidiana analizadas, solo 3 provienen de una fuente desconocida. Esto sugiere que las dos fuentes más importantes de obsidiana en el centro-sur de Chile, conocidas hasta la fecha, corresponden a la de la obsidiana negra MEL en los Nevados de Sollipulli y de la obsidiana $\mathrm{CH}$ del Volcán Chaitén. Dos de los sitios que contienen obsidiana de fuente desconocida están localizados más al norte, lo que sugiere que la fuente de estas obsidianas también podría situarse más al norte que las dos fuentes conocidas. 
Tabla 6. Elementos mayores (en \% en peso de óxidos) $y$ en trazas (ppm) en obsidianas riolíticas de fuentes del centro-sur de Chile y Neuquén, Argentina.

\begin{tabular}{|c|c|c|c|}
\hline fuente & Melipeuco & Vn Chaiten & Portada Covunco \\
\hline & (MEL) & $(\mathrm{CH})$ & (PC1) \\
\hline $\mathrm{SiO} 2$ & 73,59 & 75,6 & 75,5 \\
\hline $\mathrm{TiO} 2$ & 0,22 & 0,11 & 0,14 \\
\hline $\mathrm{Al} 2 \mathrm{O} 3$ & 13,84 & 13,78 & 13,19 \\
\hline $\mathrm{Fe} 2 \mathrm{O} 3$ & 1,84 & 1,58 & 1,23 \\
\hline $\mathrm{MnO}$ & 0,06 & 0,07 & 0,05 \\
\hline $\mathrm{MgO}$ & 0,21 & 0,22 & 0,09 \\
\hline $\mathrm{CaO}$ & 1,25 & 1,33 & 0,54 \\
\hline $\mathrm{Na} 2 \mathrm{O}$ & 4,71 & 4,16 & 4,44 \\
\hline $\mathrm{K} 2 \mathrm{O}$ & 3,95 & 3,07 & 4,71 \\
\hline P2O5 & 0,09 & & 0,02 \\
\hline LOI & 0,52 & 0,16 & 0,32 \\
\hline Total & 100,3 & 100,03 & 100,2 \\
\hline $\mathrm{Cs}$ & 5,5 & 8,6 & 8,4 \\
\hline $\mathrm{Rb}$ & 113 & 127 & 177 \\
\hline $\mathrm{Sr}$ & 134 & 148 & 46 \\
\hline $\mathrm{Ba}$ & 774 & 650 & 278 \\
\hline $\mathrm{La}$ & 21,1 & 28,3 & 33,4 \\
\hline $\mathrm{Ce}$ & 41 & 49,5 & 63,7 \\
\hline $\operatorname{Pr}$ & 4,26 & & 5,85 \\
\hline $\mathrm{Nd}$ & 15,8 & 18 & 18,7 \\
\hline $\mathrm{Sm}$ & 2,55 & 2,96 & 2,79 \\
\hline $\mathrm{Eu}$ & 0,66 & 0,53 & 0,43 \\
\hline $\mathrm{Gd}$ & 2,93 & & 3,08 \\
\hline $\mathrm{Tb}$ & 0,43 & 0,38 & 0,55 \\
\hline Dy & 2,56 & & 2,95 \\
\hline Ho & 0,57 & & 0,6 \\
\hline $\mathrm{Er}$ & 1,6 & & 1,78 \\
\hline $\mathrm{Tm}$ & 0,23 & & 0,32 \\
\hline $\mathrm{Yb}$ & 1,69 & 1,49 & 2,19 \\
\hline $\mathrm{Lu}$ & 0,3 & 0,22 & 0,36 \\
\hline $\mathrm{Y}$ & 15 & 13 & 18 \\
\hline $\mathrm{Zr}$ & 226 & 88 & 157 \\
\hline $\mathrm{Nb}$ & 7 & 9 & 29 \\
\hline Th & 11,2 & 15,8 & 26,8 \\
\hline $\mathrm{Hf}$ & 5,2 & 2,9 & 4,8 \\
\hline $\mathrm{Pb}$ & 24,3 & & 22,4 \\
\hline $\mathrm{U}$ & 3,5 & 4,3 & 7,9 \\
\hline $\mathrm{La} / \mathrm{Yb}$ & 12,5 & 19 & 15,3 \\
\hline
\end{tabular}

\section{AGRADECIMIENTOS}

Agradecemos a Serafín González y a su familia por habernos proporcionado los artefactos pertenecientes al museo de sitio del Puente Quilo, Chiloé. J. Muñoz agradece el apoyo de la Oficina Técnica Puerto Varas de SERNAGEOMIN. Las investigaciones de Christian García fueron realizadas en el marco del Proyecto Fondecyt 1060216.

\section{BIBLIOGRAFÍA}

ADÁN, L., R. MERA, M. URIBE y M. ALVARADO. 2005. La tradición cerámica bícroma roja sobre blanco en la región sur de Chile: los estilos decorativos Valdivia y Vergel. En Actas del XVI Congreso Nacional de Arqueología Chilena, editado por M. Massone, pp. 399-410. Escaparate Ediciones, Concepción.

BELLELLI, C. y F. X. PEREYRA. 2002. Análisis geoquímicos de obsidiana: distribución, fuentes y artefactos arqueológicos en el Noroeste del Chubut (Patagonia Argentina). Werken 3:99-118.

BELLELLI, C.; F. X. PEREYRA y M. CARBALLIDO. 2006 Obsidian localization and circulation in northwestern Patagonia (Argentina): sources and archaeological record. En Geomaterials in Cultural Heritage, editado por M. Maggetti y B. Messiga, pp. 241-255. Geological Society, Special Publications, London.

FERNÁNDEZ, J. 1988-1990. La cueva de Haichol. Arqueología de los pinares cordilleranos del Neuquén. Anales de Arqueología y Etnología 43/45.

GARCÍA, C. 2008. Cazadores recolectores en el área lacustre de la vertiente occidental Andina (39오): cronología, contextos y procesos. En: Arqueología de Patagonia: una mirada desde el último confín, editado por M. Salemme, F. Santiago, M. Álvarez, E. Piana, M. Vázquez y M.E. Mansur. Editorial Utopías, Ushuaia. En prensa.

GOÑI, R. 1991. Arqueología de sitios tardíos en el valle del arroyo Vilcunco (Provincia del Neuquén, Argentina). En Actas del XI Congreso Nacional de Arqueología Chilena, editado por H. Niemeyer, Tomo III: 217-226. Museo Nacional de Historia Natural, Sociedad Chilena de Arqueología, Santiago.

HAJDUK, A. 1986. Arqueología del Montículo Angostura. Primer fechado radiocarbónico. Provincia del Neuquén. Ediciones Culturales Neuquinas, Neuquén.

LÓPEZ , L.; A. PÉREZ, y C.R. STERN. 2009. Fuentes de aprovisionamiento y distribución de obsidianas en 
la Provincia del Neuquén (noroeste de la Patagonia Argentina). Intersecciones, en prensa.

MUÑOZ, J.; y C.R. STERN. 1989. Alkaline magmatism within the segment $38-39^{\circ} \mathrm{S}$ of the Plio-Quaternary volcanic belt of the southern South American continental margin. Journal of Geophysical Research, 94: 4545-4560.

MUÑOZ, J.; D. BASUALTO; H. MORENO; P. PEÑA; y M. MELLA. 2008. Geochemistry and magmagenesis of the early May 2008 rhyolitic magma erupted by Chaitén volcano, Southern Andes Volcanic Zone. Abstracts American Geophysical Union Fall Meeeting. San Francisco.

NAVARRO, X. 1984. Arqueología y Computación: un análisis de los líticos acerámicos de Quillén-1 (IX Región). Tesis para optar al grado de Licenciatura en Arqueología. Universidad de Chile.

NAVARRO, X., y M. PINO. 1995. Interpretación de ocupaciones precerámicas y cerámicas en los distintos micro ambientes de la costa de Chan Chan, Valdivia, X Región. En Actas del XIII Congreso Nacional de Arqueología Chilena 1:127-134.
NAVARRO, X., y M. PINO. 1999. Estrategias adoptivas en ambientes costeros del bosque templado lluvioso de la zona Mapuche; una reflexión desde el precerámico. En "Soplando en el Viento" Actas de las III Jornadas de Arqueología de la Patagonia, San Carlos de Bariloche, Argentina, May 1996, pp. 65-82.

STERN, C. R.; X. NAVARRO y J. MUÑOZ. 2002. Obsidiana gris translúcida del volcán Chaitén en los sitios arqueológicos de Quilo (Isla Grande de Chiloé) y Chanchán (X Región), Chile, y obsidiana del Mioceno en Chiloé. Anales del Instituto de la Patagonia Serie Cs. Hum. 30: 167-174.

STERN, C.R, X. NAVARRO, J.D. PINO, y R. VEGA. 2008. Nueva fuente de obsidiana en la Región de la Araucanía, centro-sur de Chile: Química y contexto arqueológico de la obsidiana riolítica negra de los Nevados de Sollipulli. Magallania, 36(2): 185-193.

VALDÉS, C.; M. SÁNCHEZ; J. INOSTROZA; P. SANZANA y X. NAVARRO. 1982. Excavaciones arqueológicas en el alero Quillén 1, Provincia Cautín, Chile. En: Actas del IX Congreso Nacional de Arqueología Chilena, pp.339-435. Dirección de Bibliotecas, Archivos y Museos y Sociedad Chilena de Arqueología. La Serena. 